\title{
Continuous medical education in endocrinology in Europe. A 1999 European Board of Endocrinology survey
}

\author{
H Perrild ${ }^{1}$, R Gaillard ${ }^{2}$, J W F Elte ${ }^{3}$ and R Bouillon ${ }^{4}$ \\ ${ }^{1}$ Endocrine Unit, Department of Internal Medicine, Bispebjerg University Hospital, Copenhagen, Denmark, ${ }^{2}$ Div. Endocrinologie et Metabolisme, \\ BH-19, CHUV, Lausanne, Switzerland, ${ }^{3}$ St Franciscus Gasthuis, Indwendige Geneeskunde, Rotterdam, The Netherlands and ${ }^{4}$ Lab. Experimentele \\ Geneeskunde \& Endocrinologie, Onderwijs \& Navorsing, UZ Gasthuisberg, Leuven, Belgium \\ (Correspondence should be addressed to H Perrild, Endocrine Unit, Department of Internal Medicine, Bispebjerg University Hospital, Copenhagen, \\ Denmark; Email: hans.perrild@dadlnet.dk)
}

\section{Introduction}

The European Union of Medical Specialists (UEMS), through its 34 specialist sections including the Section of Endocrinology, is in the position to know the conditions which each speciality needs to meet in order to reach harmonisation in Europe, both as regards duration and content of training and the practice of the specialist disciplines registered in the Medical Directives in each of the EU member states. The European Boards are working groups of the UEMS specialist sections and include delegates appointed by scientific and academic bodies. The Boards are in the position to define conditions which will ensure high quality training and the necessary criteria for a training centre. The Boards are further in a position to put forward suggestions regarding methods of assessment for individuals, if they so desire.

The UEMS Section of Endocrinology and the European Board of Endocrinology (EBE) have issued 'Definition of endocrinology and outline of training needs of endocrinologists' (Leuven 1991) in the UEMS document 'Compendium on medical specialist training in endocrinology in the EC' (1992). Charters on content and length of training in endocrinology (and the relation to a common trunk) and training centres were issued in 1995. In 1996 'Proposal for classification and training durations of specialities registered in doctors directives' was issued and forwarded to the EU and national authorities to influence the considerations on revision of the medical directives.

\section{Recognition of training centres}

The EBE has, in the past year, finalised the recognition of 130 training centres for endocrinology all over Europe, according to the set of rules decided by the Section and the Board and published in the European Journal of Endocrinology (1999 138 233). The basic principle in the offer to the training centres is that quality assessment and quality assurance, especially at the European level, should be a moral obligation and a voluntary process conducted by the profession. It is the view of the UEMS that the competent body to define the training of medical specialists at the national level is, and should be, a national authority taking advice from professional organisations, and setting rules in accordance with national rules and EU legislation for training and training centres, but it is hoped that the recommendations of the UEMS are considered.

\section{European exchange of trainees in endocrinology}

The list of EBE recognised centres will soon be made public both in writing and on the website of UEMS. The first possible effect of this initiative will be the introduction of sponsored fellowships for trainees in endocrinology who want to go from one centre to another for a period in their training programme. They will, of course, have to ensure that this education counts in the nationally recognised programme.

\section{Recognition of quality in endocrinology}

The next step for the EBE has been to award individual recognition of quality in endocrinology (RQE). This can be seen as the first initiative to create a voluntary, individual continuous medical education (CME) system on a European level. In the first instance, it was decided by the Board to ask the teachers at the training centres to apply for RQE. A minimum of 3 years post specialisation is required and also a listing of CME activities, very similar to the UEMS list of activities that is part of the suggested CME credit point system. The individual RQE applications have now been evaluated by the national representative on the EBE, together with an independent EBE representative from another country. In all, 500 people have applied and have been approved and they will now receive the official approval, a specially designed certificate with the UEMS logo, and the signature of the president and secretary of the Board of Endocrinology.

The offer to apply for RQE for endocrinologists that are not teachers at recognised centres will now be 
made public, following approval of the application form at the general assembly of the EBE at the last meeting in May 1999 in Lisbon. Application was free until March 2000. Since then a fee is required in order to support the secretariat that will have to deal with this enormous task. The Board has decided that this task, if possible, should be handled within the framework possible from the current financial support to UEMS Section of Endocrinology and EBE from member states, and from the fee, which should be reasonably small.

\section{Continuous medical education and continuous professional development}

Continuous professional development (CPD) is the mechanism by which the trained physicians keep updated to meet the needs of their patients. CPD includes the continuous acquisition of new knowledge, skills and attitudes. There is no sharp division between CME and CPD. CME is considered by the UEMS to be a moral obligation. The continuous updating of knowledge and skills has happened in the past and it will happen in the future, but the CME effort of the medical profession is often hidden. It is the view of the UEMS and also of the EBE that there seems to be little need to make CME mandatory (but this can be decided at the national level), but that we must respond to a mounting pressure from outside for a more visible CME in the profession, a demand which is coming from patients, health insurance companies and governments.

CME has to be structured and evaluated at the national level. At the European level and in the UEMS Specialist Sections Boards one of the goals is to harmonise the different speciality-based systems of awarding credits that are being developed in many places. Another goal is to stimulate the national CME providers to develop programmes that can be attended by more European endocrinologists looking for quality CME. One current initiative from the UEMS is the suggestion to form a European Accreditation Council for CME (EACCME), with the officers of the management council of the UEMS and a national representative from each member country. The aim is to make CME credits transparent and transferable (reciprocity within Europe), to create an umbrella structure for the affiliated professional CME authorities and a clearing-house for accreditation of CME providers in Europe. It is not intended to be a control organisation, but to ensure that the UEMS committees get an overview. The Boards, the national specialist organisations and the national authorities shall continue doing their job, and the EACCME will have close co-operation with these institutions, both nationally and internationally. Describing the CME goals, and creating a network with a knowledge of and stimulating reciprocity of the awarding of CME credits between nations, should encourage doctors to participate in international CME and in collecting information on their individual CME. The UEMS suggestion is to harmonise a credit point system and, specifically, to encourage the use of the 'hour of CME' or equivalent as the unit of measurement for credit points, to ensure implementation on a greater scale. The UEMS suggestion is that 250 points should be gained over 5 years with a distinction between internal and external points (100 of each). Further information and details can be found on the website www.uems.be/ cme.htm

\section{European survey on continuous medical education in endocrinology (CMEE) in 1999}

Members of the education committee of the European Board of Endocrinology (comprising Hans Perrild (Denmark), Franco Sanchez-Franco (Spain), Catherine Waeber (Switzerland), George Tolis (Greece) and Jan Willem Elte (The Netherlands)) have collected current information from all EU member states on the present state of CME in endocrinology. The national members of the EBE and the UEMS Section of Endocrinology were, during 1997 and 1998, asked questions about CME at the national level: whether CME was obligatory or not, whether there had been established national CME data collecting bodies, CME boards and programmes (possibilities listed: universities, professional organisations, scientific organisations, medical associations or journals), a national CME point system and evaluation system and whether re-certification is a consequence of lacking information on CME and, lastly, to what degree CME is paid for by industrial sponsors. All national delegates present at the last general assembly of the EBE and the UEMS Section of Endocrinology in Lisbon in May 1999 were asked to go through the details once more before publication.

\section{Obligatory or voluntary CME}

CME in endocrinology is now obligatory in two countries (The Netherlands and Switzerland) and expected to be in another three (Austria, Spain and Greece) (Table 1). CME has been obligatory in France since 1996 , but is not yet regulated by the government. It is organized by the profession and universities. In Italy, we are told it has been mandatory since 1992 but it has never been implemented. From another UEMS survey we know that formal CME is considered necessary by all countries except Finland (1997). We know that legislation for obligatory CME is present in Austria and The Netherlands. In Denmark, all hospital based specialists in endocrinology have a formal right to attend 10 days of CME paid for by their employers, but it is not obligatory to attend CME in Denmark. 
Table 1 Obligatory or voluntary CME in UEMS member countries in 1999.

\begin{tabular}{lll}
\hline & & Obligatory \\
\cline { 2 - 3 } & General & Endocrinology \\
\hline Austria & No* & No* \\
Belgium & Yes & No \\
Denmark & No & No \\
Finland & No & No \\
France & Yes** & Yes** \\
Germany & No* & No \\
Greece & No & No* \\
Ireland & No* & No \\
Italy & No*** & No \\
Luxembourg & No & No \\
Netherlands & Yes & Yes \\
Norway & No & No \\
Portugal & No & No \\
Spain & No & No* \\
Sweden & No & No \\
Switzerland & Yes & Yes \\
United Kingdom & No & No \\
& &
\end{tabular}

*Planned to be obligatory. **Compulsory by law since 1996 , but not yet regulated by the government. ${ }^{* * *}$ Plans for mandatory CME under consideration.

\section{National CME bodies, data collecting bodies and programmes}

CME data collecting bodies are present in 8/17 countries and managed by the profession in most countries except Belgium, where the national insurance organisation is the data collecting body (Table 2 ). Seven out of seventeen countries have a national CME body and four of these also have a national CME body in endocrinology (Table 3). Ten out of seventeen countries have no national CME programme, but seven have or are planning to have one (Austria, Belgium, Denmark, Germany, Greece, The Netherlands, Switzerland) (Table 4).

\section{Credit point systems}

Seven countries have created a credit point system for endocrinology (Austria, Belgium, Greece, Ireland, The Netherlands, Switzerland, United Kingdom).

The credit points (cp) are collected yearly in Ireland (50 cp) and the United Kingdom (50 cp), every three years in Belgium (200 cp per year) and Austria (only in general medicine), and every five years in The Netherlands (28 cp per year). Credit points are awarded for participation in national/international meetings/seminars, typically 1 point/h (Belgium gives $5-10$ points/h but has a higher final total number). Talking at meetings is awarded 10 points, talks to students 1 point. Being a course leader is awarded 10 points (in Belgium 20). A visit to an endocrine training centre is worth 8 points. Between 15 and 5 points are awarded for publications depending on whether the recipient is the first or second author (in Belgium 20 ( $\max 40$ )). Reading current literature is worth $6-15$ points and participation in courses or activities within ethics or economics is worth $10-15$ points.

\section{Industrial involvement}

CME was paid for/sponsored by industry in 8/17 countries (from 50-95\%), 5 countries did not respond. Five out of seventeen countries indicated that CME was organised by industry in $5-20 \%$ of cases.

\section{Recertification}

The UK indicated a possibility of loss of certificate if no CME was undertaken. In The Netherlands authorisation might be lost and in Belgium CME points had an influence on reimbursement.

Table 2 National CME data collecting body and/or CME evaluation organiser established in UEMS member countries in 1999.

\begin{tabular}{ll}
\hline Austria & Austrian medical association \\
Belgium & National insurance organisation (RIZIV) \\
Denmark & No \\
Finland & No \\
France & - \\
Germany & Medical association (evaluation: Ministry of Health) \\
Greece & Royal College of Physicians \\
Ireland & No information \\
Italy & No \\
Luxembourg & Netherlands Association of Internal Medicine (NIV) \\
Netherlands & No \\
Norway & Medical association and universities \\
Portugal & No \\
Spain & No \\
Sweden & Medical association (FMH) \\
Switzerland & Royal College of Physicians \\
United Kingdom &
\end{tabular}


Table 3 National general CME board established in UEMS member countries in 1999. Asterisk indicates national CME board for endocrinology.

\begin{tabular}{ll}
\hline Austria & No \\
Belgium & Universities, professional organisations, medical associations \\
Denmark & No \\
Finland & Council National de Formation Continue Medicin \\
France & Professional organisations ( ${ }^{*}$ scientific organisations: endocrine and diabetes) \\
Germany & Professional organisations, scientific organisations, medical associations ( ${ }^{*}$ same) \\
Greece & Royal College of Physicians \\
Ireland & No information \\
Italy & No \\
Luxembourg & NIV + scientific organisations and medical associations ( ${ }^{*}$ scientific organisations: endocrine and diabetes) \\
Netherlands & No \\
Norway & No \\
Portugal & No \\
Spain & FMH national association $\left({ }^{*}\right.$ scientific organisations: endocrine) \\
Sweden & No \\
Switzerland &
\end{tabular}

\section{Conclusion}

In this survey, The European Board of Endocrinology has documented the activity in CME already in progress in many of the UEMS member countries. The EBE thinks that it is important to make its position on CME in endocrinology clear now, especially if the profession wants to have an impact on a forthcoming European CME system that will not only ensure a voluntary system but also attempt to harmonise the national programmes and evaluation systems. This will ensure that, in the future, European endocrinologists can collect $\mathrm{CME}$ points for their national CME evaluation at accredited courses both nationally and internationally. The EBE has therefore decided to issue the EBE position on CME in endocrinology in Europe, as published here. It is hoped that this will serve as an inspiration and a guideline for the national endocrine professional organisations and national authorities.

\section{Acknowledgements}

The executive committee of The European Board of Endocrinology 1998-2002 is composed of Professor R Bouillon (president), Professor R Gaillard (secretary, treasurer), Professor Thomopoulos (vice president), Nicolas Guéritée (honorary secretary) and associate Professor Hans Perrild (CME and chairman of education committee).

Table 4 National general CME programme established in UEMS member countries in 1999. Asterisk indicates national CME programme for endocrinology.

\begin{tabular}{ll}
\hline Austria & Yes ( ${ }^{*}$ yes) \\
Belgium & Yes, Ministry of Health and Social Affairs \\
Denmark & In preparation \\
Finland & No \\
France & Yes, Bundesärtzekammer (yes, scientific societies: endocrine and diabetes) \\
Germany & Yes, Ministry of Health \\
Greece & Under review \\
Ireland & No \\
Italy & More or less, medical associations (NIV) ( ${ }^{*}$ same) \\
Luxembourg & No ( ${ }^{*}$ yes, scientific societies: endocrine) \\
Netherlands & - \\
Norway & No \\
Portugal & No ( ${ }^{*}$ yes, scientific societies: endocrine) \\
Spain & In preparation ( ${ }^{*}$ in preparation) \\
Sweden & No \\
Switzerland & \\
United Kingdom &
\end{tabular}




\section{The position of the European Board of Endocrinology on CME}

Each of the specialist sections of the European Union of Medical Specialists has set up a specialist Board whose mandate includes not only the encouragement and maintenance of good standards of training but also the stimulation and co-ordination of continuing education after training is completed.

In the Charter on Continuing Medical Education of Medical Specialists (Harmonisation Committee, UEMS, June 1994) it says: "The task of the European Boards includes the provision of advice and stimulation, at a European level, in relation to: setting of standards, assessment and accreditation, quality assurance, safeguarding financial independence and European co-ordination of programmes.'

The aim of continuous medical education (CME) for the European endocrinologist is to practice within the existing guidelines, advise and guide the patient, educate and train, evaluate scientific work and be knowledgeable in ethical matters.

The European Board of Endocrinology (EBE) supports the position stated by the UEMS, that CME is a moral obligation and that the national programme for CME is essential and should be supported and encouraged. CME should be visible but voluntary. We further support the position that CME should, at all times, be independent of commercial financial interests, and finally it should be organised and evaluated by the profession in co-operation with relevant authorities.

The European Board of Endocrinology encourages the specialist associations in endocrinology, diabetes and metabolism to establish CME programmes and a CME evaluation system, at the national level, in co-operation with authorities conducting postgraduate education and evaluation.

This national CME body and evaluating system in endocrinology, diabetes and metabolism shall create a local credit point system. It is recommended that this credit point system follows the recommendations for CME credit points set up by UEMS, in order to harmonise the credit point system in Europe. The European Board of Endocrinology endorses the idea that providers of international meetings forward applications to the European Accreditation Council (EAC) for CME credit point accreditation, an accreditation delegated by EAC to the EBE. We further encourage providers of national meetings to seek international accreditation for their meetings, thereby encouraging doctors to participate in international CME. The European Board of Endocrinology will regularly list national meetings of interest for CME on the forthcoming website.

The European Board of Endocrinology shall not collect the credit points from individual doctors, but will ask for the awarded credit points when individual doctors apply for recognition of quality in endocrinology (RQE).

In order to be awarded recognition of CME, EBE is of the opinion that it should be documented that the applicant is seeing patients a certain minimum number of hours a week.

The European Board of Endocrinology recommends the use of the 'hour of CME' as the unit of measurement. It recommends the collection of 250 points over 5 years: 100 of these from formally planned interactive CME, suitable for international credit (conferences, meetings, courses, study in approved institutions, all accredited CME points), 100 of these from personal learning activities (peer consultation, small group discussion, journal clubs, medical writing, lectures, seminars not designated as formal CME, development and review of quality assessment data, use of clinical data bases). On top of this, a certain number of hours reading literature should be included ( $2 \mathrm{~h}$ per week).

The European Board of Endocrinology judges the collection of points from planned interactive CME to be the main focus, but is not in favour of individual reporting of a detailed log of personal learning activities. Therefore, at the 5-year evaluation the latter should be reported as a yes or no, 100 points reached. We do, however, stress the importance of including in personal learning activities ethics (attitudes) as an important future CME activity. It is also mandatory that the CME should be balanced within the different areas of the speciality.

The European Board of Endocrinology has started stimulating CME activity in the endocrine profession in Europe by offering individual doctors a possibility to apply for an RQE certificate, to be awarded every 10 years to endocrinologists having passed their ninth postgraduate year.

This text and other related data can be found at the UEMS website www.uems.be/cme.htm

European Board of Endocrinology, September 1999. 